\title{
EDITORIAL
}

\section{Problemas de salud mental en la sociedad: Un acercamiento desde el impacto del COVID 19 y de la cuarentena}

\author{
Carmen Cecilia Caballero-Domínguez ${ }^{1}$ (D), Adalberto Campo-Arias ${ }^{2}$ (iD
}

1. Universidad del Magdalena. Santa Marta, Colombia. Correo electrónico: ccaballero@unimagdalena.edu.co - https://orcid.org/0000-0003-3730-2750

2. Universidad del Magdalena. Santa Marta, Colombia. Correo electrónico: acampoa@unimagdalena.edu.co - https://orcid.org/0000-0003-2201-7404

La sociedad mundial vive una experiencia sin precedente, que moviliza todas las estructuras gubernamentales, sanitarias y sociales. Desde diciembre de 2019 a 15 de abril de 2020, la pandemia de coronavirus (COVID-19) se ha propagado a 213 países, con 1.848 .439 casos confirmados en el contexto mundial y 676.162 en América Latina. En lo transcurrido del 6 de marzo hasta 15 de abril, en Colombia se informaron 3.105 casos y 131 muertes $^{1}$.

La Organización Mundial de la Salud ${ }^{2}$ sugirió el aislamiento social, limitación de la movilidad o cuarentena de la población, como la estrategia más efectiva en la contención y mitigación de la velocidad de propagación de la infección y evitar el colapso de los sistemas estatales de salud. No obstante, el distanciamiento social genera reacciones adaptativas y desadaptativas ${ }^{3,4}$. Las condiciones que generan el aislamiento social se configuran como un estresor no normativo que incrementa la posibilidad presentar por primera vez problemas mentales o la exacerbación o recurrencia de trastornos mentales preexistentes ${ }^{5}$.

Las reacciones psicológicas ante una pandemia suelen ser agudas; sin embargo, es posible observar secuelas emocionales a largo plazo ${ }^{3,6}$. Estas reacciones dependen de ciertas características individuales, como la intolerancia a la incertidumbre, la vulnerabilidad percibida a la enfermedad o la propensión a la ansiedad ${ }^{3}$. El estrés emocional tiene consecuencias neurobiológicas que incrementan la probabilidad de exacerbar enfermedades concomitantes y la vulnerabilidad a reunir criterios para un trastorno mental, en particular, trastornos de ansiedad, depresivos, del sueño y estrés agudo ${ }^{5,7}$. Asimismo, la preocupación exagerada por la salud se presenta en diferentes niveles de intensidad, con significaciones e interpretaciones, generalmente, erróneas y catastróficas de las sensaciones corporales, habitualmente ignoradas ${ }^{5}$.

Además, el temor por el contagio, la desinformación, la distorsión o el exceso de información pueden causar preocupaciones confusión, ira o frustración. A ello se le debe sumar el aburrimiento por el aislamiento, las dificultades o pérdidas financieras que genera la situación y la preocupación por el estigma-discriminación, en caso de ser positivo para la infección o, paradójicamente, ser trabajador del sector salud encargado de cuidados directos a pacientes convalecientes ${ }^{2,4,8,9}$.

Es necesario destacar el efecto del contagio de la pandemia en los familiares del personal de salud en quienes pueden presentarse no sólo las preocupaciones esperables ante la cercanía con personas convalecientes de sus parejas, hijos o hermanos, sino también síntomas depresivos o de estrés postraumático, como reexperimentación, alteraciones negativas en la cognición e hiperexcitación ${ }^{6,10}$. Por otra parte, el personal de la salud en la primera línea de la atención de pacientes gravemente enfermos por contagio del COVID-19, son igualmente vulnerables a problemas de salud mental, debido a la muerte de pacientes, el exceso de horas y presiones laborales y el distanciamiento de familiares, en algunos $\operatorname{casos}^{11}$. El personal de salud puede presentar síntomas depresivos, incluso ideación suicida o suicidio, síntomas de trastornos relacionados con estrés 
agudo o manifestaciones inespecíficas de sufrimiento emocional ${ }^{10,11}$. Por ejemplo, médicos en Canadá manifestaron malestares como miedo, aislamiento social y estrés laboral ${ }^{12}$, y en China, los médicos que atendieron los primeros casos de COVID-19 informaron síntomas relacionados con trauma emocional ${ }^{10,13}$. De la misma manera, personal de enfermería en China refirieron síntomas de traumatización indirecta, dada a la empatía e identificación con la vulnerabilidad y dolor del paciente; estas personas acusaron pérdida de apetito, fatiga, deterioro físico, trastornos del sueño, irritabilidad, falta de atención, entumecimiento, miedo y desesperación ${ }^{6}$.

La política pública colombiana, sin bien establece la salud como un derecho de la sociedad y promueve la atención médica primaria y atención de salud mental, estos lineamientos se deben concretar en acciones efectivas y verificables para garantizar el derecho a la salud integral. El sistema de salud requiere estructurar servicios psicológicos para intervención en crisis ${ }^{8,14}$, programas de salud mental dirigidos a mitigar el trauma y estrés en los profesionales sanitarios ${ }^{6}$, capacitar al personal en atención primaria para mitigar la ansiedad en los pacientes y familia ${ }^{8}$; asimismo, implementar programas de apoyo social dirigidos a realizar acciones de prevención y manejo de la creciente ola de estigma-discriminación asociado al COVID$19^{15}$.

Las medidas de salud públicas para la mitigación y contención de pandemia requieren de un esfuerzo intersectorial (educación, medios de comunicación, salud, sociedad y ciudadanos) dirigidos a modificar actitudes, creencias y comportamientos que permita la adopción de medidas de autocuidado responsable, en pro del bien personal y comunitario. De la misma forma, adoptar e incorporar la tecnología como un recurso para el asesoramiento psicológico e intervención en crisis, teléfono e Internet, para los profesionales asistenciales, pacientes, familiares y sociedad en general, como estrategia para mitigar el impacto emocional y facilitar el retorno a la vida habitual en condiciones seguras, una vez se supera el período de crisis $^{14,16}$.

\section{REFERENCIAS BIBLIOGRÁFICAS}

1. Ministerio de Salud y Protección Social. Situación actual: Nuevo Coronavirus (Covid 19) reporte. 15-04-2020-Corte 8:00pm. https://www.minsalud.gov.co/salud/publica/PE T/Paginas/Covid-19_copia.aspx

2. Brooks S, Webster R, Smith L, Woodland L, Wessely S, Greenberg N, Rubin JG. The psychological impact of quarantine and how to reduce it: rapid review of the evidence. Lancet. 2020; 395: 912-20. Doi: http://dx.doi.org/10.1016/ S0140-6736(20)304

3. Taylor S. The psychology of pandemics: Preparing for the next global outbreak of infectious disease. New Castle: Cambridge Scholars Publishing; 2019.

4. Rubin J, Wessely S. The psychological effects of quarantining a city. BMJ. 2020; 368: 313. Doi: http://dx.doi.org/org/10.1136/bmj.m313

5. Asmundson G, Taylor S. How health anxiety influences responses to viral outbreaks like COVID-19: What all decision-makers, health authorities, and health care professionals need to know. Anxiety Disord. 2020; 71: 102211. Doi: http://dx.doi.org/10.1016/j.janxdis.2020.10221 1

6. Li Z, Ge J, Yang M, Feng J, Qiao M. Jiang R, Bi J. Vicarious traumatization in the general public, members, and non-members of medical teams aiding in COVID-19 control. Brain Behav Immunity. In Press. Doi: http://dx.doi.org/10.1016/j.bbi.2020.03.007

7. Vahia I, Blazer D, Smith G, Karp J, Steffens D, Forester B, et al. COVID-19, mental health and aging: $A$ need for new knowledge to bridge science and service. Am J Geriatr Psychiatry. In Press. Doi: http://dx.doi.org/10.1016/j.jagp.2020.03.007.

8. Duan L, Zhu G. Psychological interventions for people affected by the COVID-19 epidemic. Lancet Psychiatry. 2020; 7(4): 300-2. Doi: 
http://dx.doi.org/10.1016/S2215-0366 (20)

30073-0.

9. Lima C, Medeiros P, Carvalho L, Lima I, Nunes J., Saraiva J, et al. The emotional impact of coronavirus 2019-Ncov (New Coronavirus Disease). Psychiatry Res. 2020; 287: 112915.

Doi:

http://dx.doi.org/10.1016/j.psychres.2020.112 915

10. Lui N, Zhang F, Wei C, Jia Y, Sang Z, Sun L, et al. Prevalence and predictors of PTSS during COVID-19 outbreak in China hardest-hit hreas: Gender differences matter. Psychiatry Res.

2020; 16: 287. Doi:

http://dx.doi.org/10.1016/j.psychres.2020.112 921.

11. Koh D, Lim K, Chia E, Ko S, Quian F, Ng V, et al. Risk perception and impact of Severe Acute Respiratory Syndrome (SARS) on work and personal lives of healthcare workers in Singapore: what can we learn? Med Care. 2005; 43: 676-82.

12. Maunder R, Lancee W, Rourke S, Hunter J, Goldbloom D, Balderson M, et al. Factors associated with the psychological impact of severe acute respiratory syndrome on nurses and other hospital workers in Toronto. Psychosom Med. 2004; 66 (6): 938-942. Doi: http://dx.doi.org/10.1097 / 01.psy.0000145673.84698.18

13. Joob B, Wiwanitkit V. Traumatization in medical staff helping with COVID-19 control. Brain Behav Immun. 2020; (20) 30356-1. Doi: http://dx.doi.org/10.1016/j.bbi.2020.03.020

14. Xiang $Y$, Yang $Y$, Li W, Zhang L, Zhang Q, Cheung T, Ng C. Timely mental health care for the 2019 novel coronavirus outbreak is urgently needed. Lancet Psychiatry. 2020; 7(3): 228-229. Doi: http://dx.doi.org/10.1016/S22150366(20)30046-8

15. Bao Y, Sun Y, Meng S, Shi J, Lu L. 2019-nCoV epidemic: address mental health care to empower society. Thelancet.com Vol 395 February 22, 2020.

16. Yao H, Chen L. Zu Y. Rethinking online mental health services in China during the COVID19epidemic. Asian J Psychiatry. 2020; 50: 102015. Doi: http://dx.doi.org/10.1016/j.ajp 